Supporting Information

\title{
Leveraging Peptide Sequence Modification to Promote Assembly of Chiral Helical Gold Nanoparticle Superstructures
}

Soumitra Mokashi-Punekar, ${ }^{\dagger,}$ Sydney C. Brooks, ${ }^{\dagger}{ }_{\S}$ Camera D. Hogan, ${ }^{\dagger}$ and Nathaniel L. Rosi $^{*}, \dagger, *$

†Department of Chemistry, University of Pittsburgh, Pittsburgh, Pennsylvania 15260, United States

$\$$ Department of Chemical and Petroleum Engineering, University of Pittsburgh, Pennsylvania 15260, United States

$\S$ These authors contributed equally to this manuscript 

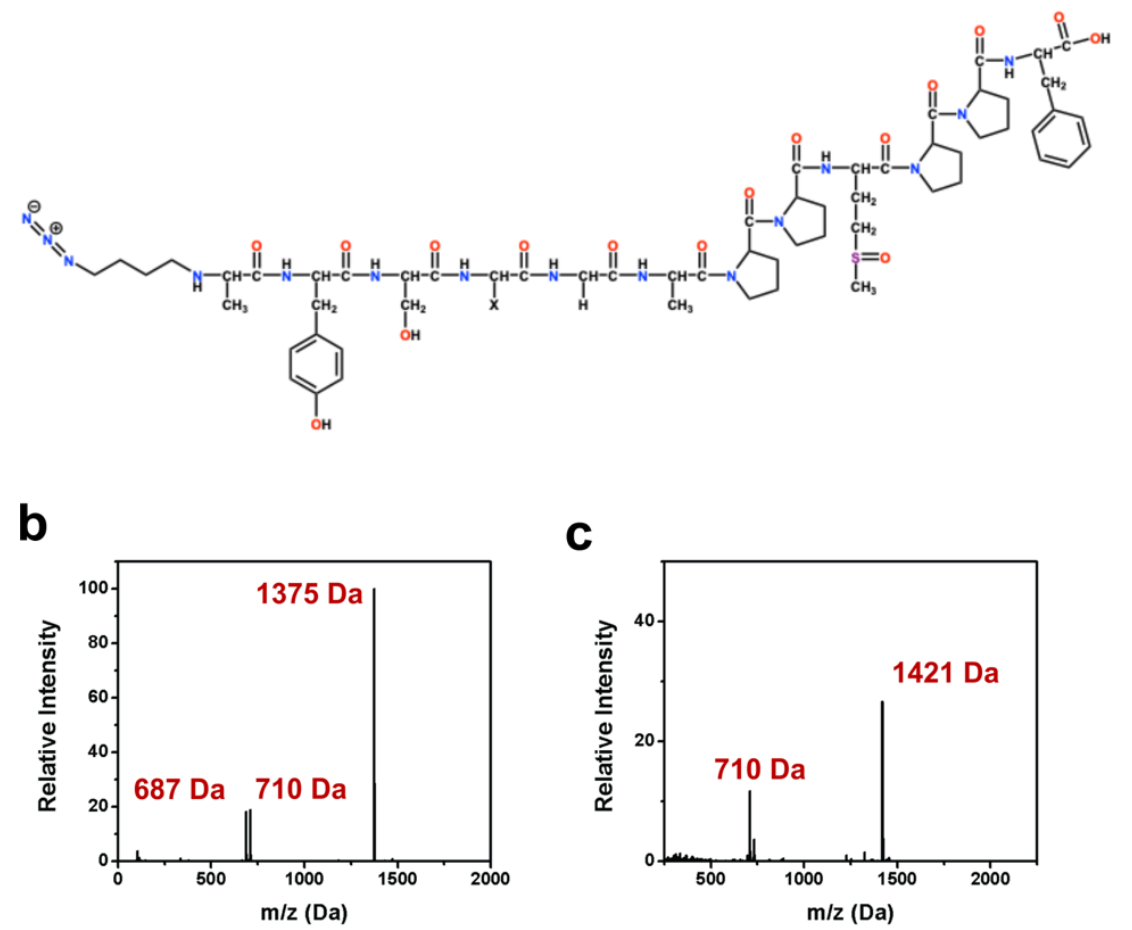

Figure S1. (a) Representative molecular structure of $\mathrm{N}_{3}-\mathrm{PEP}_{\mathrm{Au}}{ }^{\mathrm{Mox}, \mathrm{X}}$. LC-MS mass assignment of (b) $\mathrm{N}_{3}-$

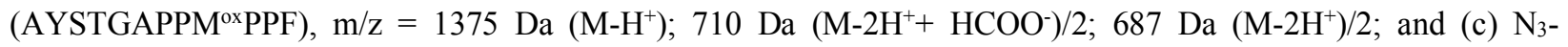
(AYSFGAPPM $\left.{ }^{\text {ox }} \mathrm{PPF}\right), \mathrm{m} / \mathrm{z}=1421 \mathrm{Da}\left(\mathrm{M}-\mathrm{H}^{+}\right) ; 710 \mathrm{Da}\left(\mathrm{M}-2 \mathrm{H}^{+}\right) / 2$. 
a
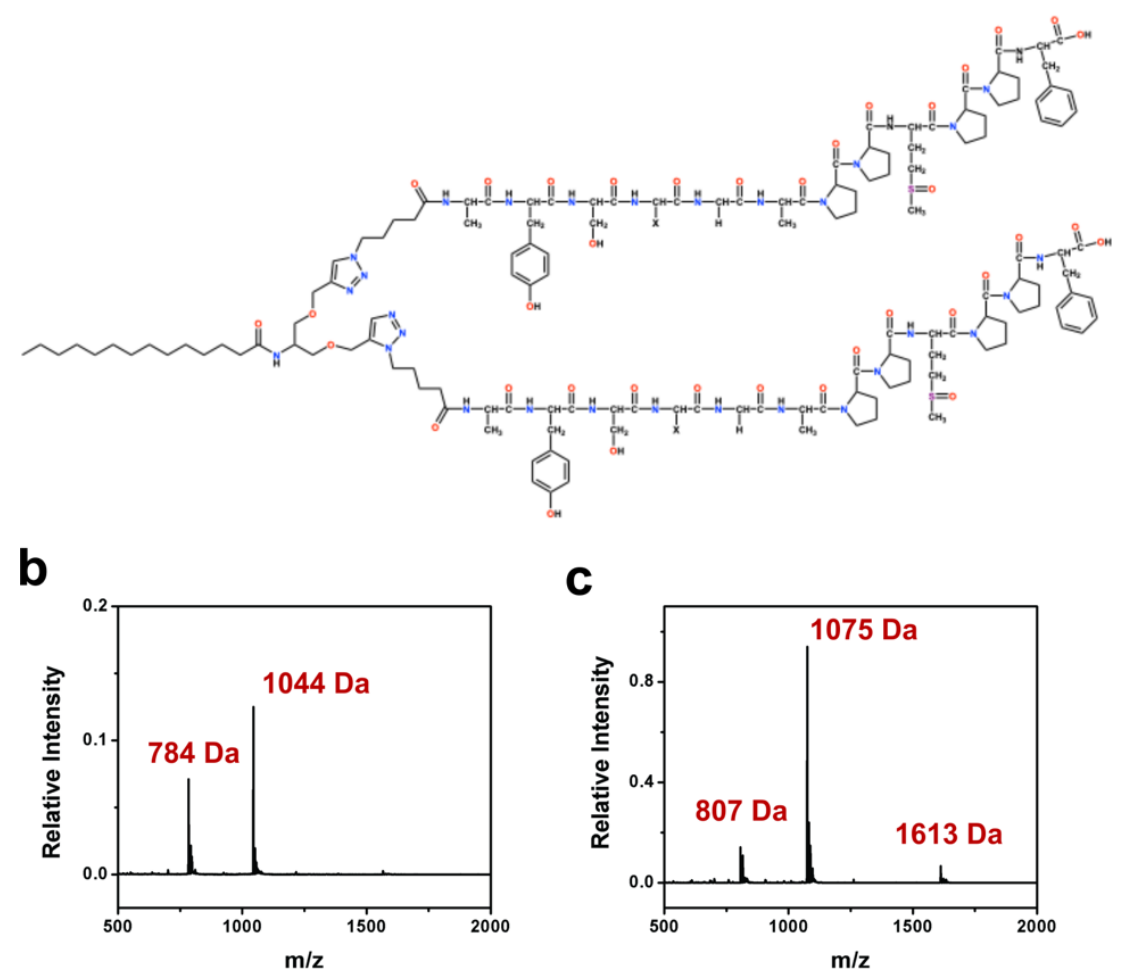

Figure S2. (a) Representative molecular structure of $\mathrm{C}_{14}-\left(\mathrm{PEP}_{\mathrm{Au}}{ }^{\mathrm{Mox}}\right)_{2}{ }^{\mathrm{X}}$. LC-MS mass assignment of (b) $\mathrm{C}_{14-}$

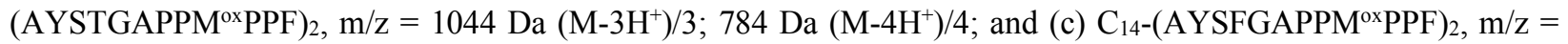
$1613 \mathrm{Da}\left(\mathrm{M}-2 \mathrm{H}^{+}\right) / 2 ; 1075 \mathrm{Da}\left(\mathrm{M}-3 \mathrm{H}^{+}\right) / 3 ; 807 \mathrm{Da}\left(\mathrm{M}-4 \mathrm{H}^{+}\right) / 4$. 


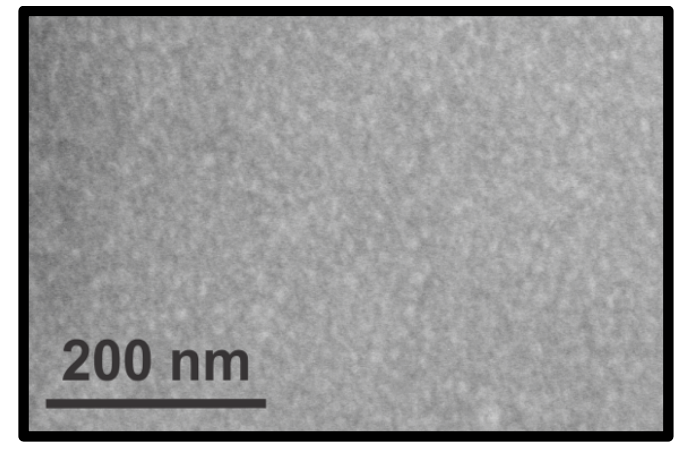

Figure S3. Negatively-stained TEM image of $\mathrm{C}_{14-}\left(\mathrm{AYSSGAPPM}{ }^{\mathrm{ox}} \mathrm{PPF}\right)_{2}$ after assembly experiment in 0.1 M HEPES. Fiber assemblies are not observed. 

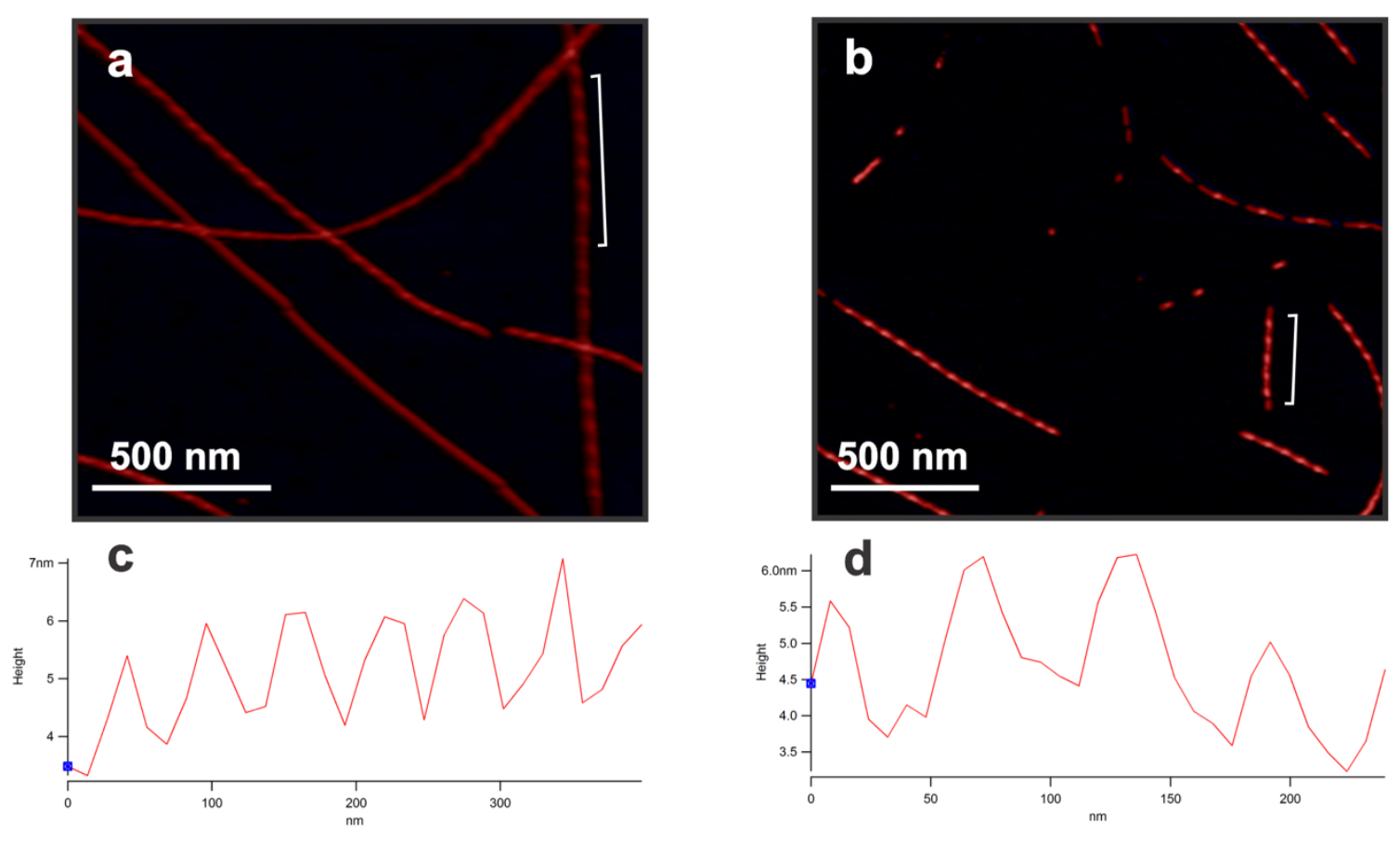

Figure S4. Additional AFM images of (a, b) $\mathrm{C}_{14}-\left(\mathrm{AYSFGAPPM} \mathrm{MxPPF}_{2}\right.$, with labeled segments corresponding to (c, d) height traces.
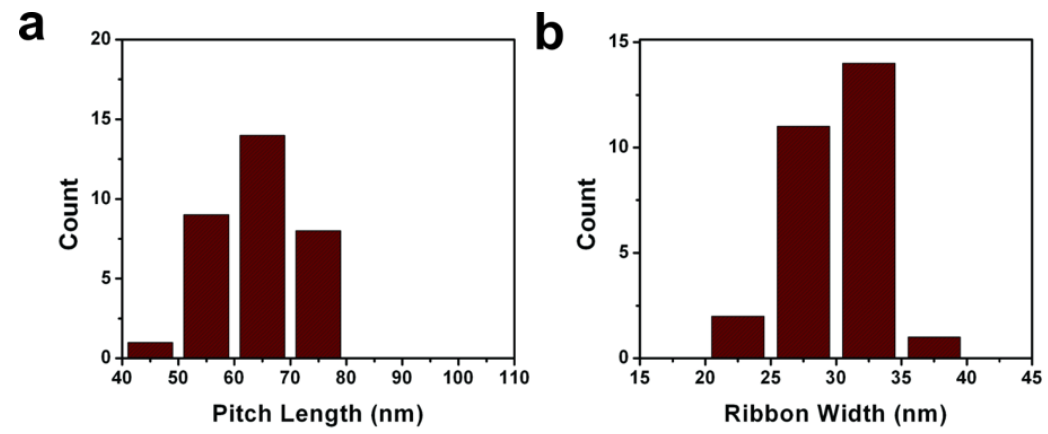

Figure S5. AFM measurements of $\mathrm{C}_{14-}\left(\mathrm{AYSFGAPPM}^{\text {ox }} \mathrm{PPF}\right)_{2}$. (a) Ribbon pitch distribution and (b) ribbon width distribution. 


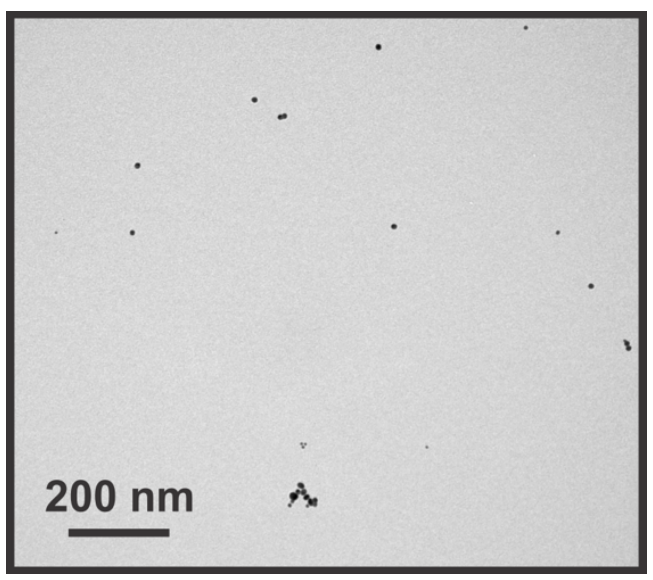

Figure S6. TEM image of discrete AuNPs synthesized using C $14-\left(A Y S S G A P P M^{\text {ox }} P P F\right)_{2}$. 

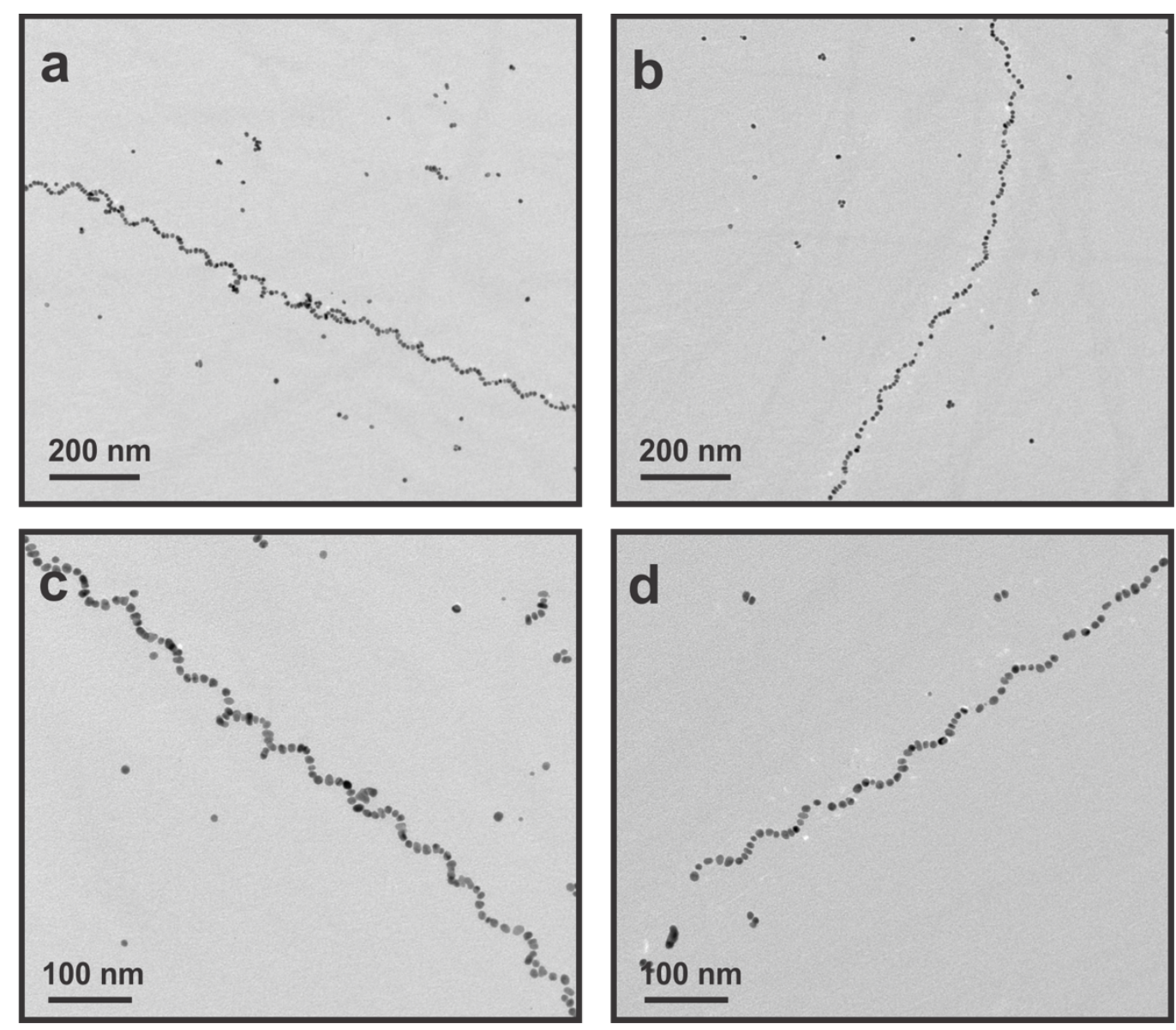

Figure S7. Additional TEM images of single helices prepared using $\mathrm{C}_{14}-\left(\mathrm{AYSFGAPPM} \mathrm{M}^{\text {oxPFF}}\right)_{2}$.

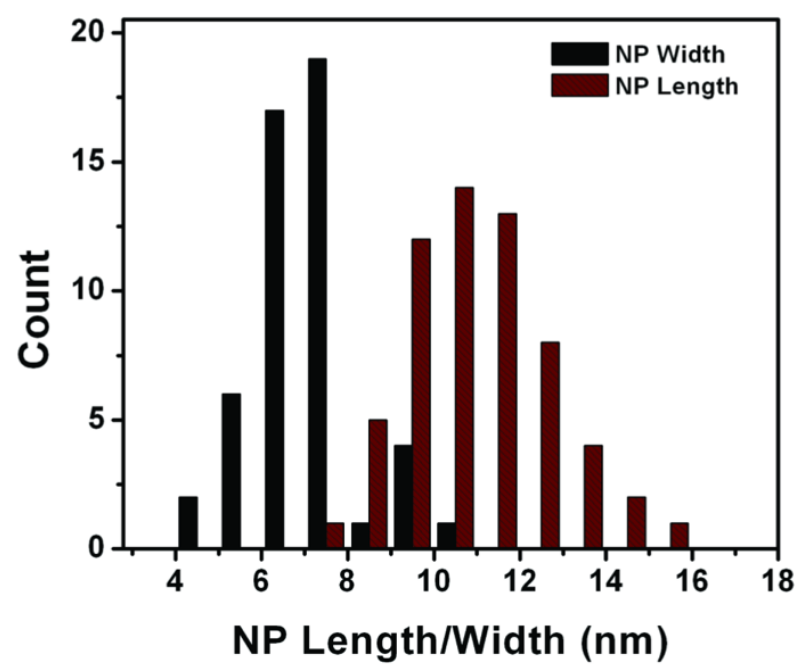

Figure S8. Particle dimensions of AuNPs in single helices prepared using $\mathrm{C}_{14}-\left(\mathrm{AYSFGAPPM}^{\mathrm{ox}} \mathrm{PPF}\right)_{2}$. 


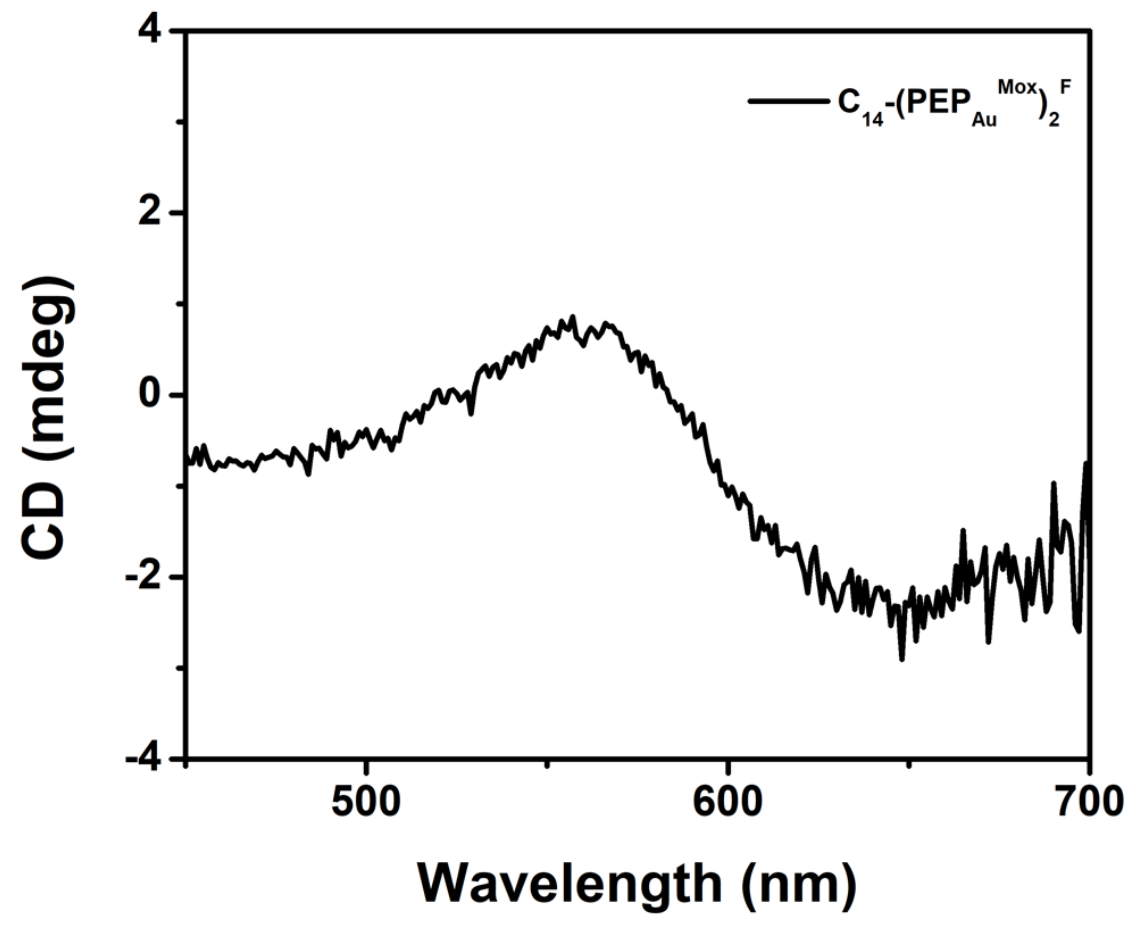

Figure S9. CD spectrum of single helices prepared using $\mathrm{C}_{14}-\left(\mathrm{AYSFGAPPM}{ }^{\mathrm{ox}} \mathrm{PPF}\right)_{2}$ showing the plasmonic chiroptical signal at $\sim 565 \mathrm{~nm}$. 


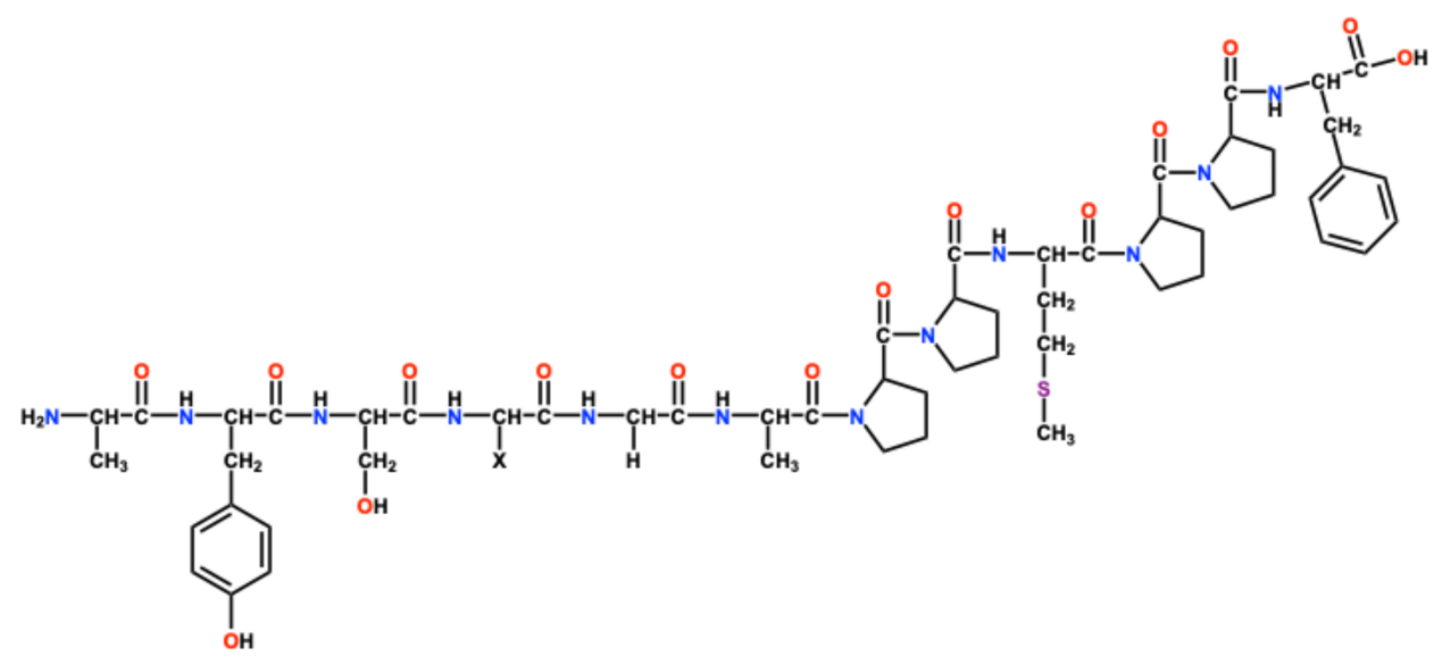

b

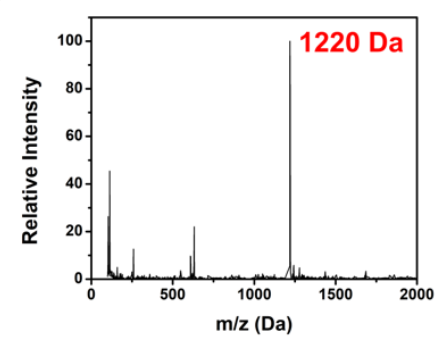

C

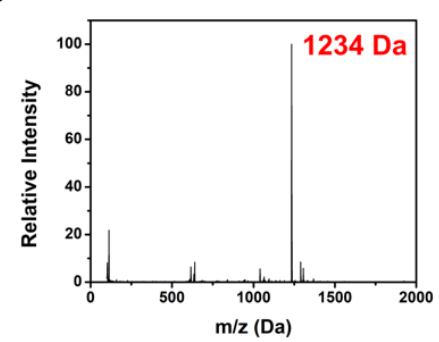

d

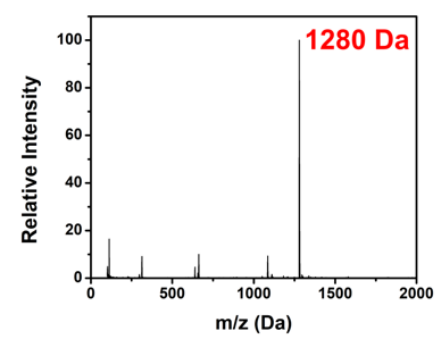

Figure S10. (a) Representative molecular structure of $\mathrm{NH}_{2}-\mathrm{PEP}_{\mathrm{Au}} \mathrm{x}$. LC-MS mass assignment of (a) $\mathrm{NH}_{2}-$ AYSSGAPPMPPF, m/z = 1220 Da $\left(\mathrm{M}-\mathrm{H}^{+}\right) ; 633$ Da $\left(\mathrm{M}-\mathrm{H}^{+}+\mathrm{HCOO}-\right) / 2 ; 609$ Da $\left(\mathrm{M}-2 \mathrm{H}^{+}\right) / 2$; (b) $\mathrm{NH}_{2-}$ AYSTGAPPMPPF, m/z $=1234 \mathrm{Da}\left(\mathrm{M}-\mathrm{H}^{+}\right) ; 641 \mathrm{Da}\left(\mathrm{M}-\mathrm{H}^{+}+\mathrm{HCOO}-\right) / 2 ; 617 \mathrm{Da}\left(\mathrm{M}-2 \mathrm{H}^{+}\right) / 2$; and (c) $\mathrm{NH}_{2}-$ AYSFGAPPMPPF, m/z = $1280 \mathrm{Da}\left(\mathrm{M}-\mathrm{H}^{+}\right) ; 663 \mathrm{Da}\left(\mathrm{M}-\mathrm{H}^{+}+\mathrm{HCOO}-\right) / 2 ; 639 \mathrm{Da}\left(\mathrm{M}-2 \mathrm{H}^{+}\right) / 2$. 


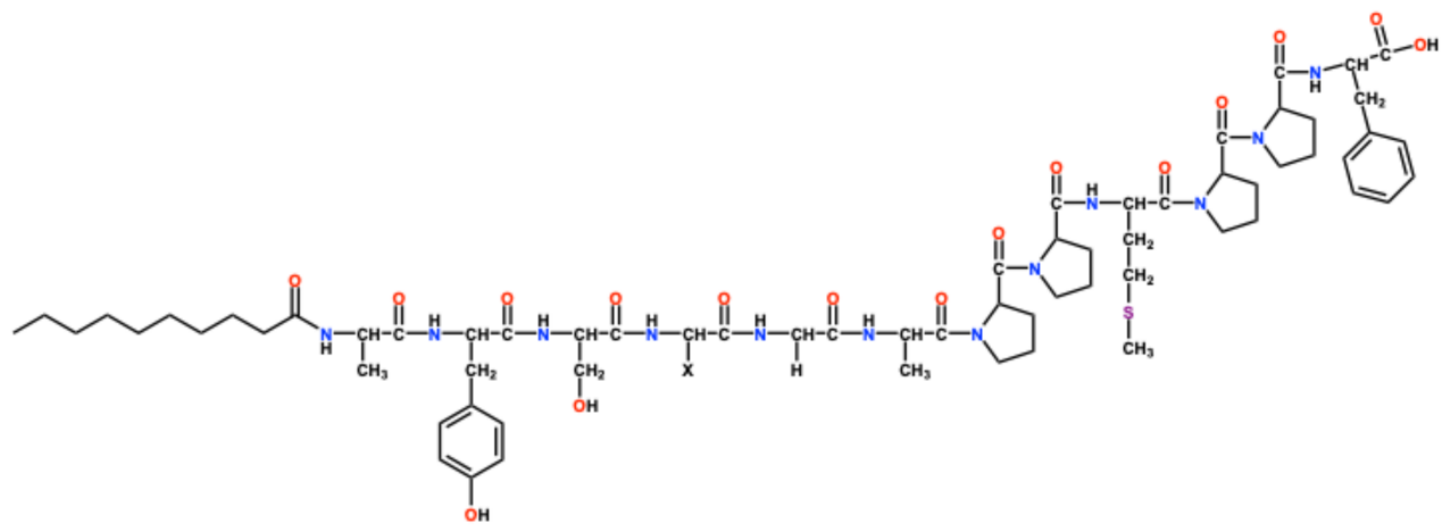

b

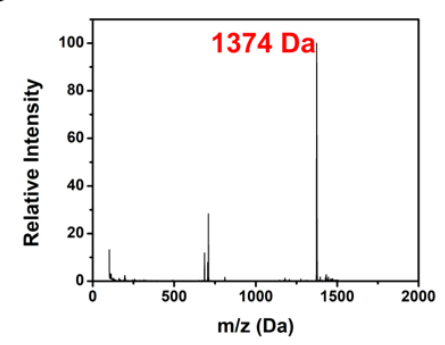

C

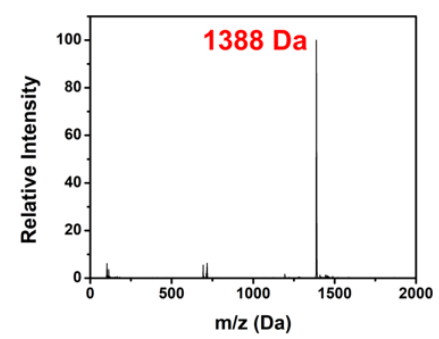

d

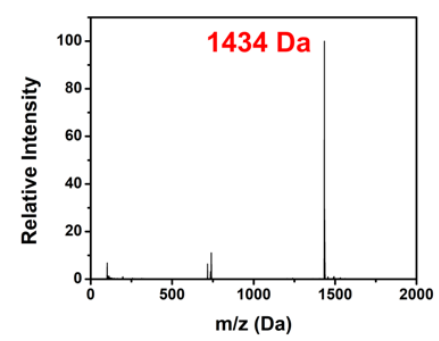

Figure S11. (a) Representative molecular structure of $\mathrm{C}_{10}-\mathrm{PEP}_{\mathrm{Au}}{ }^{\mathrm{x}}$. LC-MS mass assignment of (b) $\mathrm{C}_{10-}$

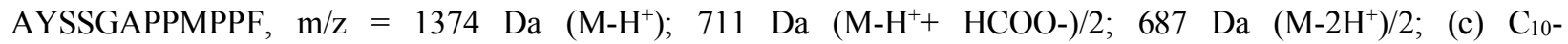
AYSTGAPPMPPF, $\mathrm{m} / \mathrm{z}=1388 \mathrm{Da}\left(\mathrm{M}-\mathrm{H}^{+}\right) ; 717 \mathrm{Da}\left(\mathrm{M}-\mathrm{H}^{+}+\mathrm{HCOO}-\right) / 2 ; 693 \mathrm{Da}\left(\mathrm{M}-2 \mathrm{H}^{+}\right) / 2$; and (d) $\mathrm{C}_{10}-$ AYSFGAPPMPPF, m/z = $1434 \mathrm{Da}\left(\mathrm{M}-\mathrm{H}^{+}\right) ; 741 \mathrm{Da}\left(\mathrm{M}-\mathrm{H}^{+}+\mathrm{HCOO}-\right) / 2 ; 717 \mathrm{Da}\left(\mathrm{M}-2 \mathrm{H}^{+}\right) / 2$. 

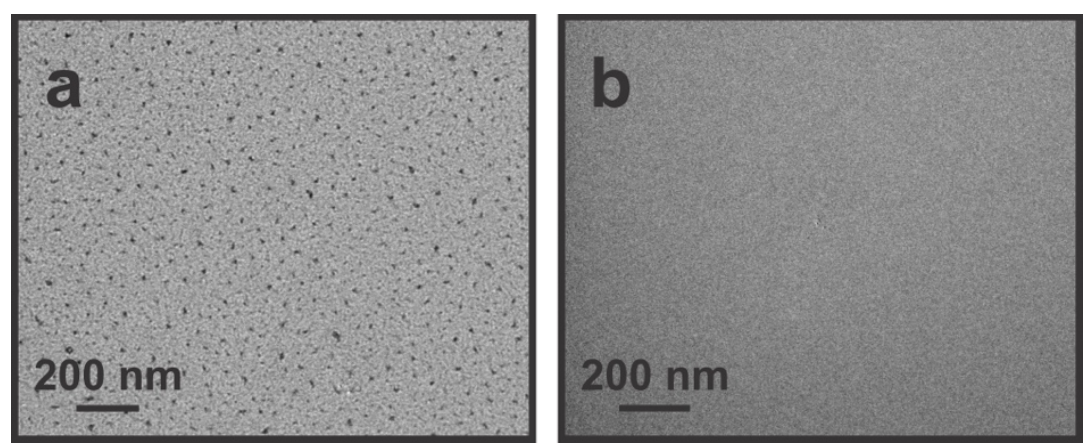

Figure S12. Negatively-stained TEM image of (a) $\mathrm{C}_{10}$-AYSSGAPPMPPF and (b) $\mathrm{C}_{10}$-AYSTGAPPMPPF after assembly experiments in 0.1 M HEPES. Fiber assemblies are not observed.
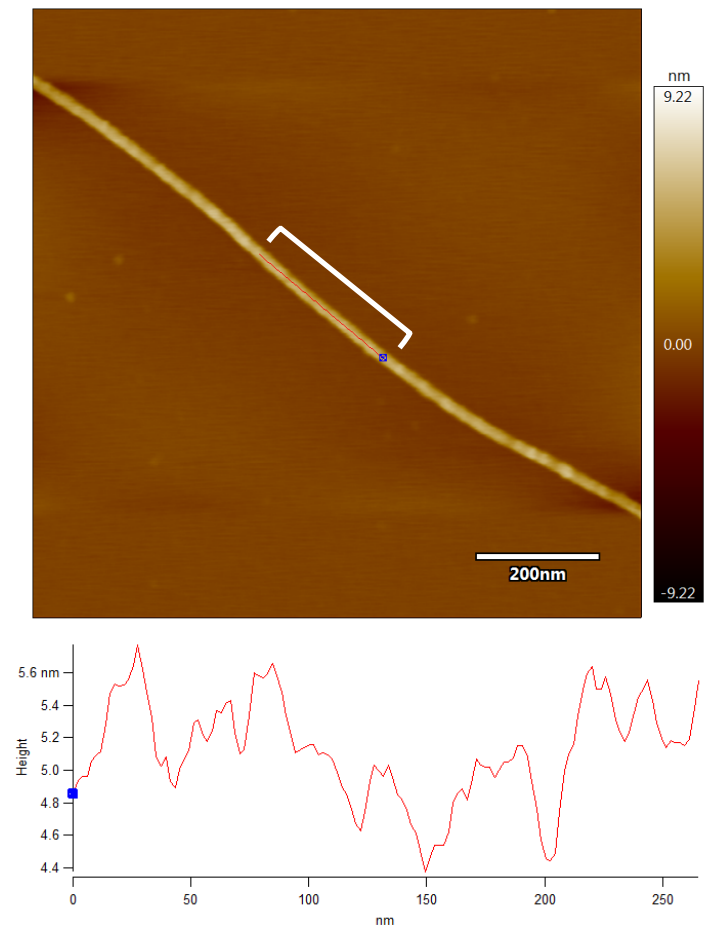

Figure S13. Additional AFM image of $\mathrm{C}_{10}$-AYSFGAPPMPPF fibers. Height trace does not indicate well-defined helicity. 

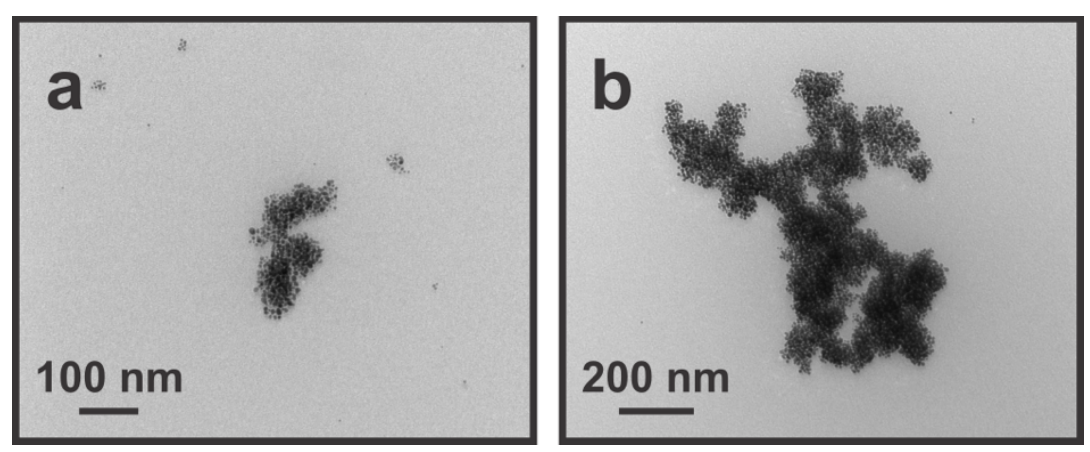

Figure S14. TEM images of discrete AuNPs/AuNP aggregates formed using (a) $\mathrm{C}_{10}$-AYSSGAPPMPPF and (b) $\mathrm{C}_{10-}$ AYSTGAPPMPPF.
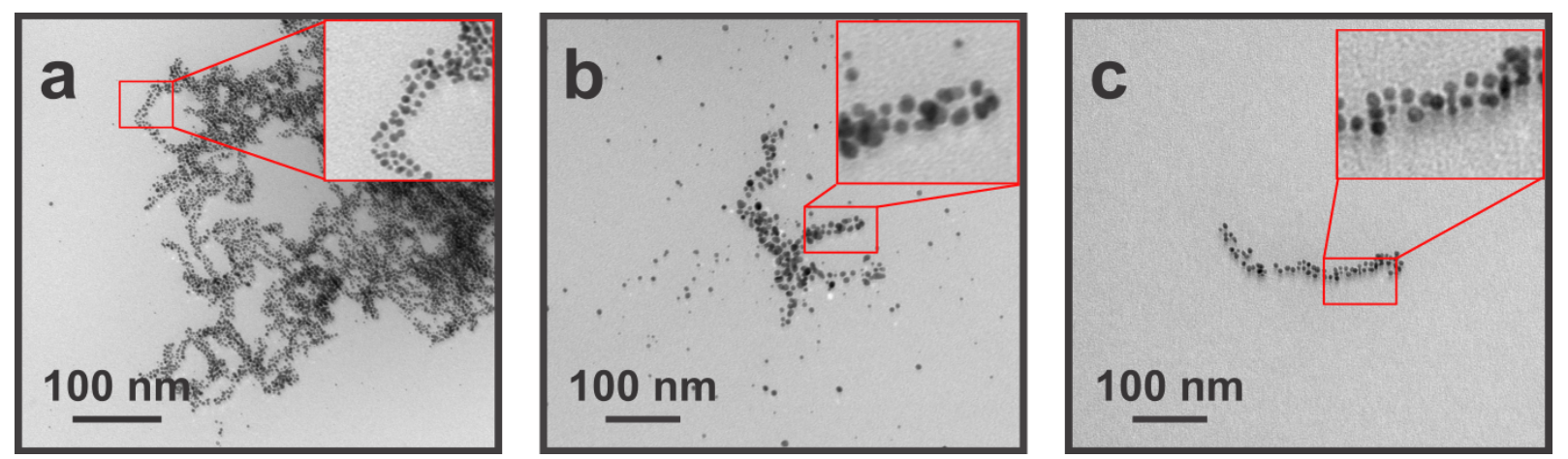

Figure S15. (a-c) Additional TEM images of AuNP assemblies formed using $\mathrm{C}_{10}$-AYSFGAPPMPPF. High magnification images highlight the helical nature of the superstructures. 


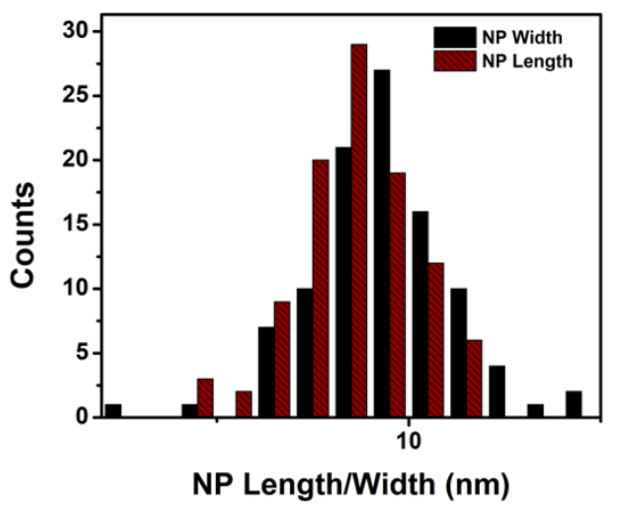

Figure S16. Particle dimensions of AuNPs within double helices formed using $\mathrm{C}_{10}$-AYSFGAPPMPPF. 